Proc. 3rd Conf. on the Intersections Between Particle and Nuclear Physics

Rockport, Maine

May $14-19,1988$
$C 015=560514=272$

BNL- -41494

TI88 $0 I 585 I$

\title{
SPECTROMETER RESULTS FROM BNL E802
}

E-802 Collaboration, Brookhaven National Laboratory, Upton, NY 11973 USA ANL-BNL-Columbia-Hiroshima-INS-Kyushu-MIT-UCBerkeley-UCRiversideTokyo

T. ABBOTT $j$, Y. AKIBA ${ }^{e}$, D. ALBURGER ${ }^{b}$, D. BEAVIS ${ }^{b}$, R.R. BETTS ${ }^{a}$, L. BIRSTEIN ${ }^{b}$, M.A. BLOOMER ${ }^{h}$, P.D. BOND ${ }^{b}$, C. CHASMAN ${ }^{b}$, Y.Y. CHU ${ }^{b}$, B.A. COLE $^{h}$, J.B. COSTALES ${ }^{h}$, H.J. CRAWFORD ${ }^{i}$, J.B. CUMMING ${ }^{b}$, R. DEBBE ${ }^{b}$, E. DUEK ${ }^{b}$, J. ENGELAGE ${ }^{g}$, S.Y. FUNG ${ }^{j}$, L. GRODZINS ${ }^{h}$, S. GUSHUE ${ }^{b}$, H. HAMAGAKI ${ }^{e}$, O. HANSEN ${ }^{b}$, R.S. HAYANO ${ }^{k}$,

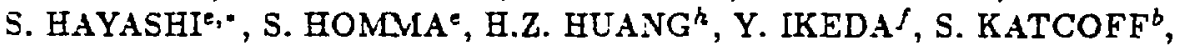
S. KAUFMAN ${ }^{a}, K$. KLMURA $^{f}$, K. KITAMURA ${ }^{d}, K$. KURITA $^{c}$, R.J. LEDOUX ${ }^{\dot{n}}$, M. J. LEVINE ${ }^{b}$, Y. MIAKE ${ }^{b, * * *}$, R.J. MORSE ${ }^{h}$, S. NAGAMTYA ${ }^{c}$, J. OLNESS ${ }^{b}$, C.G. PARSONS ${ }^{h}$, L.P. REMSBERG ${ }^{b}$, H. SAKURAI ${ }^{k}$, M. SARABURA ${ }^{h}$, A. SHOR ${ }^{b}$, P. STANKUS ${ }^{c}$, S.G. STEADMAN ${ }^{h}$, G.S.E. STEPHANS ${ }^{h}$, T. SLGITATE ${ }^{d}$, M. TANAKA $^{b}$, M. J. TANNENBAUM ${ }^{b}$, M. TORIKOSHI ${ }^{e}$, J.H. VAN DIJK ${ }^{b}$, F. VIDEBAEK ${ }^{a}$, M. VIENT ${ }^{j}$, P. VINCENT ${ }^{b}$, V. VUTSADAKIS ${ }^{h}$, W.A. WATSON $\Pi^{b}$, H.E. WEGNER ${ }^{b}$, D.S. WOODRUFF ${ }^{h}$, Y. WU ${ }^{c}$, AND W. ZAJC ${ }^{c}$

Presented on behalf of the E-802 Collaboration by: Robert J. Ledoux

a Argonne Nationàl Laboratory, Argonne, Il 60439

b Brookhaven National Laboratory, Upton, NY 11973

- Columbia University, New York, NY 10027 and Nevis Laboratories, Irvington, NY 10533

d Hiroshima University, Hiroshima 730, JAPAN

- Institute for Nuclear Study, University of Tokyo, Tokyo 188, JAPAN

$f$ Kyushu University, Fukuoka 812, JAPAN

- Lawrence Livermore National Laboratory, Livermore, CA 94550

${ }^{h}$ Massachusetts Institute of Technology, Cambridge, MA 02139

i University of California, Space Sciences Laboratory, Berkeley, CA 94720

; University of California, Riverside. CA 92507

${ }^{k}$ University of Tokyo, Department of Physics, Tokyo 113, JAPAN

* JSPS Fellowship for Japanese Junior Scientist

*** On leave of absence from University of Tokyo

\section{ABSTRACT}

Measurements of collisions of $14.5 \mathrm{GeV} / \mathrm{c}$ per nucleon ${ }^{28} \mathrm{Si}$ ions with nuclear targets using the BNL E802 spectrometer are presented. $A$ description of the experiment and preliminary particle production ratios are discussed. 


\section{INTRODUCTION}

In this talk I would like to report the latest results from $\mathrm{E} 802$ at Brookhaven National Lab. The main objective of E802 is to measure semi-inclusive spectra from nucleus-nucleus collisions with full particle identification (PID) and large kinematic coverage. In these respects it is unique among existing relativistic heavy-ion experiments at BNL and CERN. The experiment is centered around a magnetic spectrometer, augmented with other detectors, which provide global event characterization. The first run using the spectrometer was performed in April, 1987, using $14.5 \mathrm{GeV} / \mathrm{c}$ per nucleon ${ }^{28} \mathrm{Si}$ ions obtained from the AGS. Preliminary results from this run were reported on at Quark Matter $87^{1,2}$. A subsequent run in the Fall of 1987 used ${ }^{28} \mathrm{Si}$ beams to bombard $\mathrm{Al}, \mathrm{Cu}, \mathrm{Ag}$, and Au targets. The data from this run were of greater statistics and covered a larger kinematic range. The data presented here will be limited to a discussion of particle production ratios from $\mathrm{Si}+\mathrm{Au}$ collisions.

\section{EXPERIMENTAL SETUP}

The experimental setup for E802 is shown in Figure 1. The main spectrometer has an acceptance of approximately $25 \mathrm{msr}$. The magnet can produce maximum integrated field strengths of $1.5 \mathrm{Tm}$. The spectrometer is very compact (approx. $6.5 \mathrm{~m}$ ) which allows a large fraction of the kaons to traverse the entire spectrometer before they decay. There is a second arm of the spectrom-

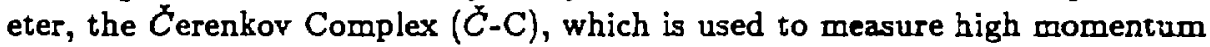
particles ( $p>4 \mathrm{GeV} / c$ ). The spectrometer can measure particles emitted with lab angles between $5^{\circ}$ and $55^{\circ}$. The acceptance of the spectrometer for pions, kaons and protons, as a function of rapidity in the nucleon-nucleon center-ofmass frame and $p_{T}$, is displayed in Figure 2. Lines of constant lab angle and momentum are plotted. The $5^{\circ}$ and $55^{\circ}$ degree lines show the limits of the angular acceptance, while the $14^{\circ}$ and $28^{\circ}$ lines illustrate the angular range subtended by one setting of the spectrometer. The lower momentum cutoff of the spectrometer is approximately $0.5 \mathrm{GeV} / \mathrm{c}$. The upper momentum lines will be discussed in connection with the limits of the PID component of the spectrometer.

The spectrometer data can be correlated with other detectors which provide both triggers and global event characterization. A minimum bias trigger is provided by beam defining scintillators just upstream of the target and a downstream scintillator (not shown in Figure 1) which measures the charge (Z) of the projectile fragment. For ${ }^{28} \mathrm{Si}$ beams, an event with a projectile fragment of $Z \leq 12$ is deemed an interaction. A hadronic calorimeter positioned at $0^{\circ}$ measures the energy contained in a $1.5^{\circ}$ cone. The target multiplicity array (TMA) measures the charged particle pseudorapidity distribution $\left(-1.2<\eta_{l a b}\right.$ $<3.0)$ and provides an online hardware trigger for central events, these being the upper $7 \%$ of the total multiplicity distribution. A 245 element $\mathrm{Pb}$-glass array is used to measure neutral energy flow in the central rapidity region ${ }^{2,3}$ 


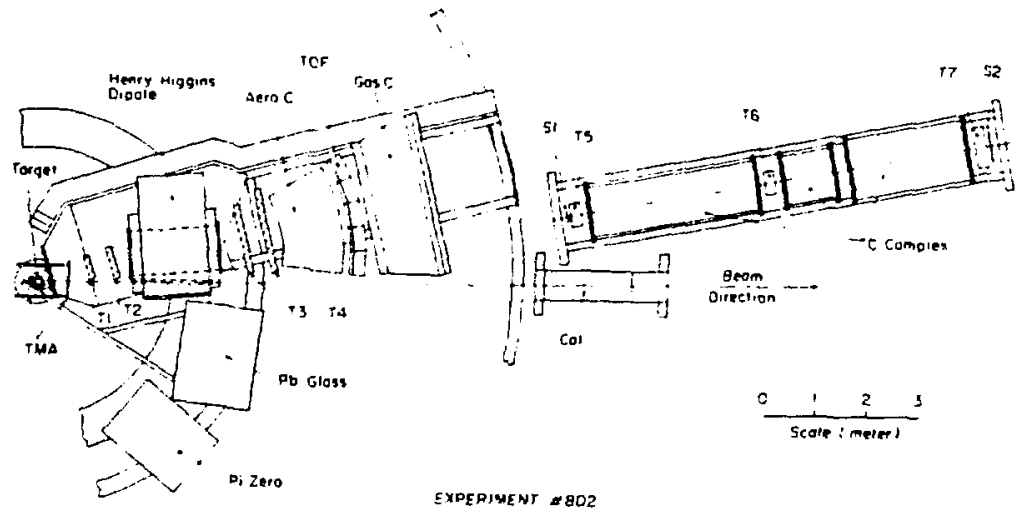

Fig. 1 Schematic view of BNL E802.

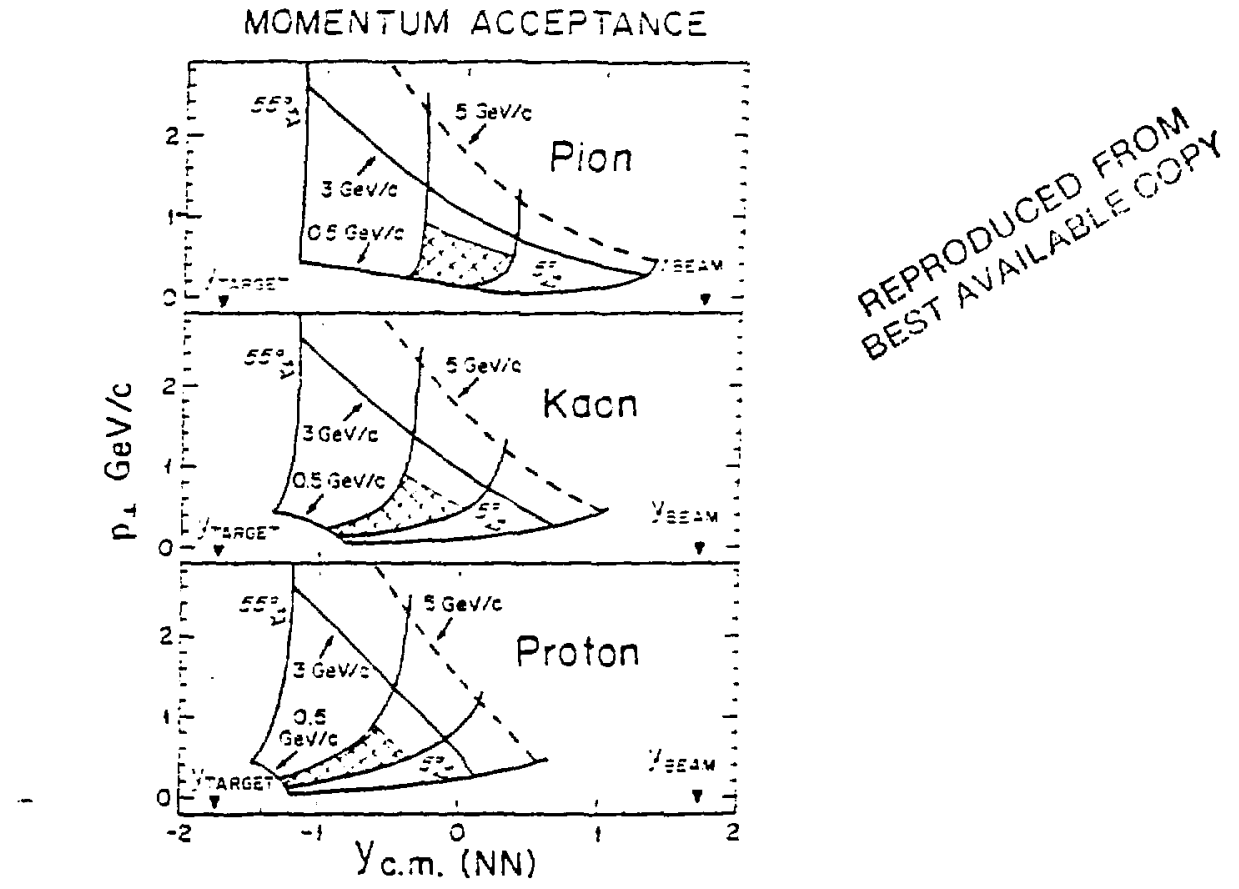

Fig. 2. Acceptance of the EBO2 spectrometer as a function of rapidity in the aucleon-nucleon center of mass same and $p_{T}$. 


$$
\left(-0.5<\eta_{c m}<0.7\right) \text {. }
$$

The large multiplicity expected in central $\mathrm{Si}+\mathrm{Au}$ collisions poses a number of technical challenges to tracking and PID. The main spectrometer was designed to handle charged particle multiplicities of up to 15 behind the magnet. This design criterion is approximately twice the predicted mean multiplicity for central $\mathrm{Si}+\mathrm{Au}$ collisions with the spectrometer at its most forward position. The analysis of the spectrometer data to date has concentrated on measurements in the central rapidity region. Thus, there has been no verification yet whether or not such high multiplicities can be properly reconstructed in the spectrometer. The measured average multiplicity behind the magnet for central $\mathrm{Si}+\mathrm{Au}$ collisions with the spectrometer spanning $14^{\circ}-28^{\circ}$ is 1-2. Less than one percent of these events have multiplicities greater than 6 behind the magnet.

The tracking and PID portions of the spectrometer were designed by exploiting some existing detector technologies, but with high segmentation and performance. The tracking of particles is accomplished with two sets of projective drift chambers before ( $\mathrm{T} 1$ and $\mathrm{T} 2$ ) and after ( $\mathrm{T} 3$ and $\mathrm{T} 4$ ) the magnet. Each set of drift chambers contains 10 wire planes with 5 views in $\mathrm{T} 1$ and 4 views in T2-T4. All wires have multi-hit capability which is provided by the use of 1ns sampling pipeline TDCs. The measured single track resolution of the drift chambers is $\sigma \approx 150 \mu \mathrm{m}$ and the double track resolution is estimated to be $\sigma \approx 2 \mathrm{~mm}$. Good single track resolution is important, since vectors formed in one chamber are used to point to another chamber. Thus, the number of vector pairs which must be considered in track reconstruction is significantly reduced as the single track resolution is improved.

PID is accomplished behind the magnet by a segmented Aerogel $\check{C}$ erenkov detector (AEROC) and a time-of-Hight scintillator array (TOF). The segmented gas $C^{\prime}$ erenkov counter (GASC) was not in place for the runs reported on here. The AEROC consists of 96 elements of Aerogel with an index of refraction of 1.02. The TOF wall consists of 160 plastic scintillator slats $(1.6 \mathrm{~cm}$ square $X$ $78 \mathrm{~cm}$ ) with a photomultiplier at both ends. The TOF resolution with software slewing correction is $\sigma \approx \mathbf{7 5}$ ps. Without slewing correction, the resolution is approximately $100 \mathrm{ps}$. The TOF wall alone allows $\pi-K$ separation at the $5-\sigma$ level for $\mathrm{p}<2.2 \mathrm{GeV} / \mathrm{c}$ and $K-p$ separation for $\mathrm{p}<3 \mathrm{GeV} / \mathrm{c}$. The AERO $\check{C}$ provides redundancy and extends the $\pi-K$ separation to $\mathrm{p}<2.8 \mathrm{GeV} / \mathrm{c}$. For the results shown here, only the PID obtained from TOF will be discussed.

\section{- PARTICLE SPECTRA}

Figure 3 is a scatter plot of $1 / \mathrm{p}$ for reconstructed tracks versus their flight time to the TOF wall. The data are from central ${ }^{28} \mathrm{Si}+\mathrm{Au}$ collisions from the Spring 1987 run, with the spectrometer spanning mid-rapidity $\left(\theta_{\text {lab }}=14^{\circ}\right.$ $28^{\circ}$ ). Comparable PD spectra were obtained in the Fall 1987 run. There are distinct particle bands for $\pi^{ \pm}, K^{ \pm}, \mathrm{p}$ and deuterons. The tracks included in the plot are those which were fully reconstructed, j.e., tracks which project back 


\section{TADLE 1.}

Preliminary particle ratios for $14.5 \mathrm{GeV} / \mathrm{c}$ per nucleon ${ }^{28} \mathrm{Si}+\mathrm{Au}$ collisions at $\theta_{l a b}=14^{\circ}-28^{\circ}$.

$$
h^{+} / \pi^{+}
$$

$$
K^{-} / \pi^{-}
$$$$
\pi^{+} / p
$$

$\mathbf{d} / \mathrm{p}$

Min. bias trigger $19 \pm 5 \%$

$5 \pm 4 \%$

$50 \pm 10 \%$

$11 \pm 3 \%$

Central Trigger $\quad 23 \pm 5 \%$

$5 \pm 4 \%$

$41 \pm 10 \%$

$9 \pm 3 \%$

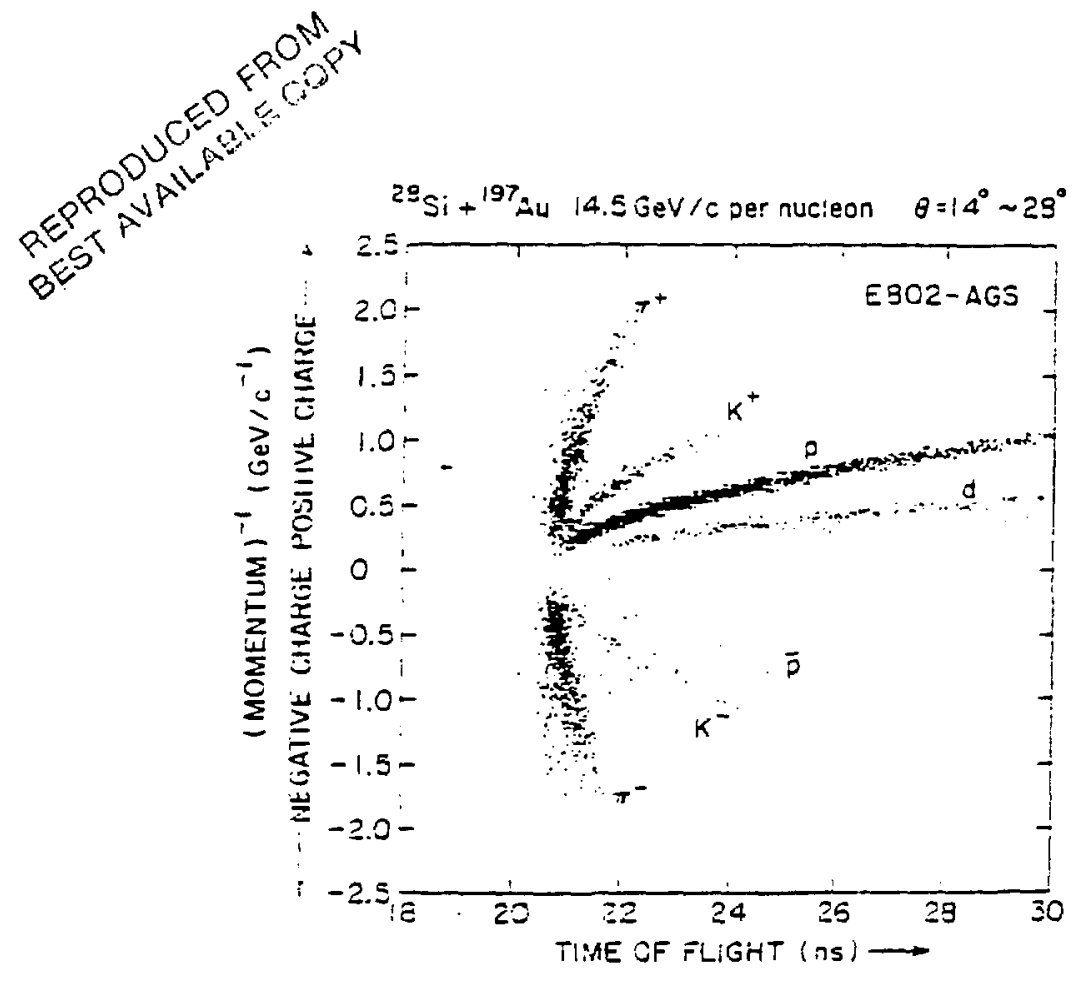

Fig. 3: Scatter plot of the inverse momentum for reconstructed tracks versus TOF for negative and positive particles from $\mathrm{Si}+$ Au ccllisions. The spectrometer spanned $\theta_{l a b}=14^{\circ}-28^{\circ}$. 
through the magnet from T3-T4 and are consistent with hits on T1-T2 and the target location. Also, only tracks which are associated with a hit on TOF and whose energy loss is consistent with $Z=1$ particles were included. Preliminary results from the April 1987 run $^{1}$ for the $K^{+} / \pi^{+}$and $K^{-} / \pi^{-}$ratios found in the region $14^{\circ}<\theta_{l a b}<28^{\circ}$ for ${ }^{28} \mathrm{Si}+\mathrm{Au}$ collisions were $19 \pm 5 \%$ and $6 \pm 5 \%$ for minimum bias events, respectively, and $24=5 \%$ and $4 \pm 4 \%$ for central events, respectively. The data are from the region indicated by the cross-hatched area in Fig. 2 and $p_{l a b}<2 \mathrm{GeV} / \mathrm{c}$. These results contain decay corrections but do not contain tracking efficiency corrections. The data were obtained using one magnet polarity. Preliminary results for $\mathrm{Si}-\mathrm{Au}$ collisions for the same angle setting for the Fall 1987 run are tabulated in Table 1. Also included in Table 1 are the $\pi^{+} / p$ and $d / p$ ratios from the Fall run. The agreement between the two runs is excellent.

Examination of Figure 2 reveals that with the spectrometer at the $14^{\circ}-28^{\circ}$ setting, the $\pi$ and $K$ come from somewhat different regions of phase space. However, by selecting particles with rapidities in the nucleon-nucleon center-ofmass frame from -0.44 to -0.14 , and combining runs of both magnet polarities, it is possible to obtain preliminary invariant cross sections as a function of $p_{T}$ which have small acceptance corrections. The $p T$ integrated $K^{+} / \pi^{+}$ratio in this restricted region is consistent with thit in Table 1.

Before discussing the significance of these particle ratios, I will briefly discuss the question of systematic errors in these measurements. It is important to note that the errors quoted in Table 1 are not indicative of the statistical error in the measurements but rather reflect an estimate of the systematic errors. Monte Carlo simulations are now under way to place the discussion of systematic errors on a quantitative footing. So far, Monte Carlo simulations which include the correct geometry and magnet map and the measured resolutions of the tracking chambers and TOF wall have been made. The Monte Carlo event generator "Fritiof" has been employed to simulate $\mathrm{Si}+\mathrm{Au}$ events. The simulated tracks are propagated through the sfectrometer and hits in the tracking chambers and TOF are "digitized". The events are then reconstructed using different parameters and cuts and compared to the input event. The $K^{+} / \pi^{+}$ and $K^{-} / \pi^{-}$ratios for input tracks with $p<2 \mathrm{GeV} / \mathrm{c}$ for $\mathrm{Si}+\mathrm{Au}$ minimum bias events in the angular range $14^{\circ}-28^{\circ}$ are $7 \%$ and $2 \%$, respectively. The reconstructed $K^{+} / \pi^{+}$and $K^{-} / \pi^{-}$ratios are $8 \pm 1 \%$ and $2 \pm 0.3 \%$, respectively. Thus, the systematic error in particle ratios from this simple Monte Carlo is on the order of $10 \%$.

\section{DISCUSSION}

The most striking feature of the particle ratios presented in Table 1 is the large $K^{+} / \pi^{+}$ratio. There is an increase of this ratio for central collision over that in minimum bias events, although both agree within the quoted errors. The $\mathrm{K}^{-} / \pi^{-}$ratio is approximately a factor of 4 smaller for both triggers. 
Since these preliminary measurements are the first of their kind in nucleusnucleus collisions, they can only be compared to results from $\mathrm{p}+\mathrm{p}$ and $\mathrm{p}+\mathrm{A}$ collisions. The $K^{+} / \pi^{+}$and $K^{-} / \pi^{-}$ratios from $19.2 \mathrm{GeV} / \mathrm{c} \mathrm{p} \div \mathrm{p}$ collisions ${ }^{5}$ are approximately $6 \%$ and $4 \%$, respectively. The available data from $p+A$ collisions are consistent ${ }^{1}$ with $\mathrm{K}^{+} / \pi^{+} \approx 10 \%$ and $\mathrm{K}^{-} / \pi^{-} \approx 4 \%$. It should be emphasized that these ratios depend on both the particle momentum and $p_{T}$. The $K^{+} / \pi^{+}$ratio as a function of $p_{T}$ for particles with $\mathrm{p}=6 \mathrm{GeV} / \mathrm{c}$ from $\mathrm{p}+\mathrm{Be}, \mathrm{Al}$ and $\mathrm{Pb}$ collisions ${ }^{6}$ at $19.2 \mathrm{GeV} / \mathrm{c}$ are shown in Figure 4. The $K^{+} / p^{+}$ratio systematically increases with target mass and $p_{T}$. The data for these measurements were from $\theta_{l a b} \approx 1^{\circ}-4^{\circ}$ and hence correspond to particles with larger center of mass rapidities than those in Table 1.

There is, of course, no substitute for measuring particle ratios for $\mathrm{p}+\mathrm{A}$ collisions in our apparatus. These measurements will be performed in the near future. Until then, it would appear that the $K^{+} / \pi^{+}$ratio in $\mathrm{Si}+\mathrm{Au}$ collision is higher than that extrapolated from $\mathrm{p}+\mathrm{A}$ data, whereas the $K^{-} / \pi^{-}$data ratio is consistent with such an extrapolation. I would like to emphasize once again that the data in Table 1 have not been corrected for tracking efficiencies.

Mechanisms have been proposed ${ }^{7}$ which qualitatively predict an increased $\mathrm{K}^{+} / \pi^{+}$ratio in relativistic nucleus-nucleus collisions. For example, in a high baryon density gas or plasma, $K^{+}$"distillation" may take place, whereby, $s \bar{s}$ pairs produced in the gas are preferentially found in the final state most often as $K^{+}$and $\Delta$ due to the large relative abundances of $u$ and $d$ quarks as compared to $\bar{u}$ and $\bar{d}$ quarks. However, it is also possible that more mundane mechanisms, such as associated production from collisions of the primary nucleons or rescattering of secondary particles in the surrounding nuclear medium could increase the production of $K^{+'}$ s. The reason for this is essentially the same, that as in a high baryon density gas, there are many available $u$ and $d$ valence quarks.

Which, if any, of the above scenarios is the most probable explanation of the measured particle ratios has yet to be determined. The data presented here are only a part of a large body of data from a variety of targets and covering a large kinematic range. We are in the process of analyzing these data and obtaining absolute cross sections. I would challenge theorists to develop models which are useful at predicting cross sections at AGS energies. There will snortly be plenty of data to explain!

\section{SUMMARY}

A fairly comprehensive set of data from collisions of $14.5 \mathrm{GeV} / \mathrm{c}$ per nucleon ${ }^{28} \mathrm{Si}$ beams with nuclear targets have been obtained using the BNL E802 spectrometer. Preliminary results from central $\mathrm{Si}+\mathrm{Au}$ collisions from two runs yieid $K^{+} / \pi^{+}$and $K^{-} / \pi^{-}$ratios for $\theta_{\text {lab }}=14^{\circ}-28^{\circ}$ (mid-rapidity) of $23 \pm 5 \%$ and $5 \pm 4 \%$, respectively. The $K^{+} / \pi^{+}$ratio is larger than that extrapolated from existing $p+A$ data. 


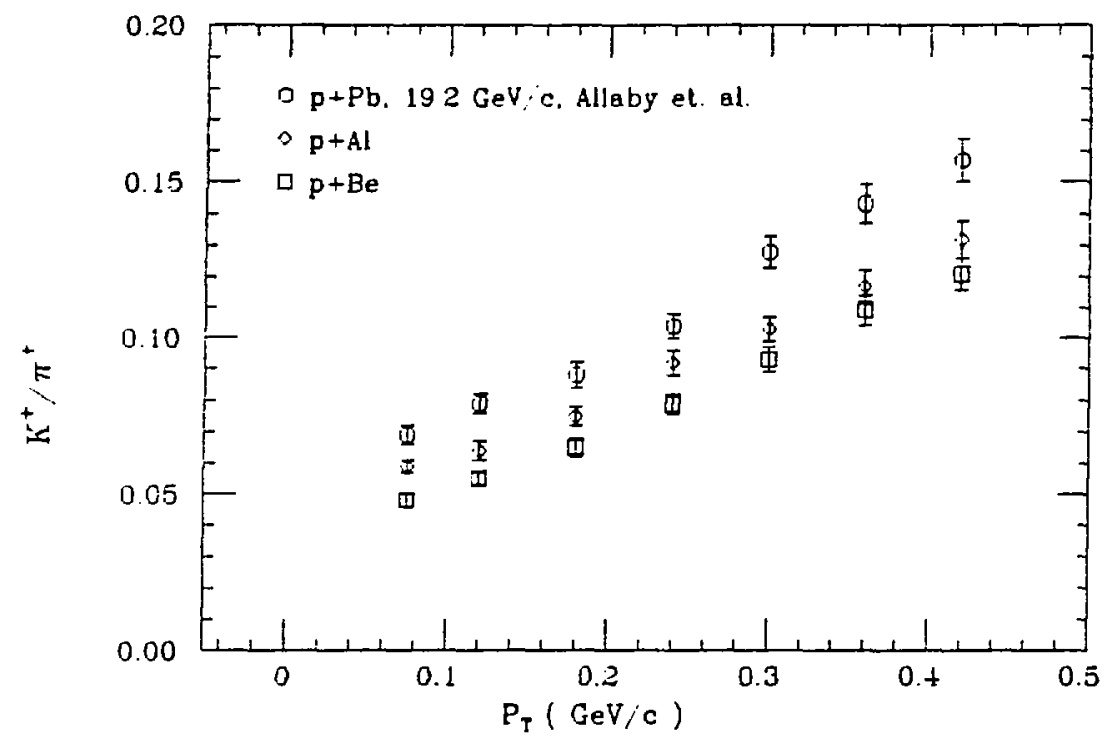

Fig. 4: The $K^{+} / \pi^{+}$ratio for $p=6 \mathrm{GeV} / \mathrm{c}$ particles for $\theta_{l a b} \approx 1^{\circ}-4^{\circ}$ as a function of $\mathrm{p}_{\mathrm{T}}$ from $19.2 \mathrm{GeV} / \mathrm{C} \mathrm{P}+\mathrm{Be}, \mathrm{Al}$ and $\mathrm{Pb}$ collisions.

\section{ACKNOWLEDGEMENTS}

E802 experiments are supported in part by the U.S. Department of Energy under contracts with ANL, BNL, Columbia, LBL, MIT, and UC Riverside, in part by the NASA through Grant NGR05-003-513 under contract with the University of California and by the US-Japan High Energy Physics Collaboration Treaty. I am indebted to all my collaborators on E802 as well as the AGS and Tandem Operation Technical Staffs.

\section{REFERENCES}

1. Y. Miake et al., Z. Phys. C38, 135 (1988).

2. L. Remsberg et al., Z. Phys. C38, 35 (1988).

3. T. Aboott et al. (E802 Collaboration), Phys. Lett. 197B, 287 (1987).

4. B. Anderson et al. Nucl. Phys. B281, 289 (1987).

5. A. N. Diddens et al., Nuovo Cimento $\underline{31}, 961$ (1964).

6. J. V. Allaby et al., CERN 70-12 (1970).

7. M. Gyulassy, Z. Phys. C38, 361 (1088). 


\section{DISCLAIMER}

This report was prepared as an account of work sponsored by an agency of the United States Government. Neither the United States Government nor any agency thereof, nor any of their employees, makes any warranty, express or implied, or assumes any legal liability or responsibility for the accuracy, completeness, or usefulness of any information, apparatus, product, or process disclosed, or represents that its use would not infringe privately owned rights. Reference herein to any specific commercial product, process, or service by trade name, trademark, manufacturer, or otherwise does not necessarily constitute or imply its endorsement, recommendation, or favoring by the United States Government or any agency thereof. The views and opinions of authors expressed herein do not necessarily state or reflect those of the United States Government or any agency thereof. 